\title{
Knockdown of GmSOG1 Compromises Drought Tolerance in Transgenic Soybean Lines
}

\author{
Samuel Aduse Poku, Peter Nkachukwu Chukwurah, Htut Htet Aung, Ikuo Nakamura* \\ Laboratory of Plant Cell Technology, Chiba University, Chiba, Japan \\ Email: ^inakamur@faculty.chiba-u.jp
}

How to cite this paper: Poku, S.A., Chukwurah, P.N., Aung, H.H. and Nakamura, I. (2021) Knockdown of GmSOG1 Compromises Drought Tolerance in Transgenic Soybean Lines. American Journal of Plant Sciences, 12, 18-36. https://doi.org/10.4236/ajps.2021.121003

Received: December 2, 2020

Accepted: January 17, 2021

Published: January 20, 2021

Copyright $\odot 2021$ by author(s) and Scientific Research Publishing Inc. This work is licensed under the Creative Commons Attribution International License (CC BY 4.0).

http://creativecommons.org/licenses/by/4.0/ (c) (i) Open Access

\begin{abstract}
Plants are regularly exposed to myriads of stress factors that cause tremendous damage to their genetic make-up. To ensure genome stability and survival over several generations under harsher environmental conditions, plants have evolved a unique mechanism for dealing with DNA damage known as the DNA damage response pathway (DDR). It has been proposed that there may exist a relationship between the DNA damage response pathway and abiotic stress response in plants. To further investigate this relationship, we knocked down the soybean suppressor of gamma response 1 gene (GmSOG1), a master regulatory gene of the DDR, in soybean plants and subjected the generated transgenic plants to drought stress analysis. Gene expression analysis of the GmSOG1 gene in drought stressed soybean tissues revealed high levels of expression in buds and young leaves. The root lengths and root fresh weights of transgenic soybean plants grown on Murashige and Skoog media supplemented with Gamborg B5 vitamins (MSB5 media) containing $200 \mathrm{mM}$ mannitol for 10 days were significantly lesser than those of drought stressed wild-type plants. Polyethylene glycol (PEG) induced drought stress assay in vivo resulted in significant damage in transgenic plants compared with wild-type plants. Also, the relative expressions of known drought responsive transcription factors such as GmDREB1 and GmLEA as well as antioxidation related genes like GmAPX and GmCAT were downregulated in transgenic soybean lines relative to wild-type plants. Moreover, wild-type soybean plants accumulated more chlorophyll and less malondialdehyde (MDA) than transgenic lines. A confirmatory experiment in GmSOG1 overexpressing Arabidopsis plants also showed significantly higher survival rates and anti-oxidation enzyme accumulation in drought stressed GmSOG1 overexpressing Arabidopsis lines compared with wild-type plants. These results suggest that the SOG1 gene may play active roles in plant abiotic stress defense.
\end{abstract}




\section{Keywords}

GmSOG1, DNA Damage, Abiotic Stress, Drought Stress

\section{Introduction}

The soybean plant is the most cultivated oil seed crop worldwide, occupying about $6 \%$ of the world's total landmass under cultivation [1]. The plant contains several useful metabolites and it is the most economically important oil seed crop as well as the leading source of vegetable oil in the world [2]. Research has identified several useful health benefits for the plant including the prevention of cancer, kidney diseases, obesity and diabetes [3].

Drought stress is one of the major factors affecting soybean production, causing about $40 \%$ of global yield losses annually [4]. Drought is usually caused by a variety of factors such as low humidity, high temperatures and water deficiency [5]. Soybean plants exposed to drought conditions experience reduced nitrogen fixation, loss of $\mathrm{CO}_{2}$ accumulation and reduction in leaf area [6], which consequently results in a decrease in protein synthesis and yield losses [7].

Given the useful nature of the soybean crop, several studies have identified useful drought defense genes in the plant. For example, Arabidopsis plants expressing the soybean dehydration responsive binding protein 2 gene (GmDREB2) were reported to have shown enhanced tolerance to drought and salt stresses [8]. The soybean ethylene responsive factor 3 (GmERF3) transcription factor was also found to confer drought tolerance to transgenic tobacco plants [9]. Wheat and Arabidopsis plants expressing the soybean basic leucine zipper 1 (GmbZIP1) transcription factor showed tolerance to drought and other abiotic stresses [10].

Under stress conditions, reactive oxygen species (ROS) such as singlet oxygen, superoxide anions and hydroxyl radicals accumulate in plants leading to DNA damage. One mechanism by which plants deal with reactive oxygen species is via the anti-oxidation pathway by means of enzymes such as catalase (CAT) and ascorbate peroxidase (APX). In addition to the antioxidation pathway, plants have evolved an efficient system known as the DNA damage response (DDR) pathway [11] [12]. The DDR pathway is made up of key components including DNA damage sensors, signal transducers, mediators, and effectors [13] that perform functions such as the activation of DNA repair pathways and the termination of cell division in proliferating cells experiencing DNA damage [14].

The suppressor of gamma response 1 (SOG1) gene has been identified as an important gene in plant DNA damage response [15]. This gene is a plant specific transcription factor belonging to the no apical meristem (NAM), Arabidopsis transcription activation factor (ATAF1/2), and cup shaped cotyledon (CUC2); NAC family of proteins [15]. In addition to a central NAC domain, the gene possesses two other domains: The $\mathrm{N}$-terminal extension and the $\mathrm{C}$-terminal transcription regulatory domain which contains five serine-glutamine sites that 
are phosphorylated in response to DNA damage [16]. The SOG1 gene has been shown to play significant roles in cell cycle regulation during DNA damage. For example, Irradiated SOG1 Arabidopsis mutants showed decreases in expression levels of genes related with cell cycle regulation such as the cyclin dependent kinase B2-1 gene (CDKB2-1) and KNOLLE for a 24-hour period [15]. Also, the growth of roots of seedlings of sog1-1 lines was not inhibited relative to those of wild-type seedlings when grown in media containing zeocin, a mutagen [17]. Other studies have also identified SOG1 to be involved in programmed cell death [18], the initiation of endoreduplication [17] and the maintenance of genome stability in response to DNA damage [15].

The ability of a plant to maintain its genome integrity under stress conditions is likely to play a vital role in stress tolerance. However, very few studies have focused on the roles DDR related genes play in abiotic stress defense [14]. Here we report the functional characterization of the GmSOG1 gene. In this work, we chose the GmSOG1 gene because of its unique characteristic as a major player in the DDR pathway. We hypothesized that the GmSOG1 gene apart from being a potentially valuable drought defense gene for soybeans, might also help us understand the interplay between the DDR and abiotic stress response in plants.

\section{Materials and Methods}

\subsection{Phylogenetic Analysis of the GmSOG1}

Amino acid sequences of the protein (XP_006604012) encoded by GmSOG1 was aligned with homologous sequences from Phaseolus vulgaris (XP_007141005), Arabidopsis thaliana (NP_001319443), Vigna unguiculata (XP_027922212), Solanum lycopersicum (XP_004231842), Capsicum annuum (XP_016561625), Gossypium hirsutum (XP_016673290), Brassica napus (XP_013642093.1) and Cucumis melo (XP_008458820) with the Clustal W software [19]. Using the MEGA 5.0 software, a phylogenetic tree was constructed by means of the neighbor-joining method. The tree was constructed with 1000 bootstrap replicates [20]

\subsection{Generation of GmSOG1 Knockdown Transgenic Soybean Lines}

A 395 bp fragment of the open reading frame of the GmSOG1 gene (XM_006603949) was PCR amplified with gene specific primers from cDNA isolated from soybean leaves. The amplified sequence was first cloned into the PCR8 entry vector, confirmed via sequencing and then cloned into the pANDA35HK RNAi vector by means of the Gateway LR cloning method. Two copies of GmSOG1 fragments were inserted in inverted orientation at both ends of the Gus linker $(1.2 \mathrm{~kb})$ sequence under the control of cauliflower mosaic virus (CaMV) $35 \mathrm{~S}$ promoter (Figure 2(B)). Half seed explants of the Japanese soybean variety "Kurosengoku" were transformed according to the method previously described by Hinchee et al. [21] with few modifications. Soybean seeds were surface sterilized with 70\% alcohol for $40 \mathrm{~s}$ and $1 \%$ sodium hypochlorite solution for 20 minutes. The steri- 
lized seeds were plated on Gamborg B5 medium and germinated for 5 days. Explants were prepared by splitting 5-day old seedlings into equal halves along the hilium to separate the two cotyledons and the axillary bud region of cotyledonary nodes wounded with a surgical blade. Agrobacterium tumefaciens EHA105 cells carrying the RNAi construct (pANDA35HK::GmSOG1i) was vacuum infiltrated into wounded explants at a pressure of $25 \mathrm{~mm}$ of $\mathrm{Hg}$ for 20 mins. Co-cultivation was carried out in the dark on MS medium overlaid with filter paper for three days. Subsequently, explants were washed, blotted dry and plated on shoot induction medium (SIM) containing $70 \mathrm{mg} / \mathrm{L}$ kanamycin for 2 weeks. Developing shoots were excised from cotyledons and transferred onto rooting medium without kanamycin. The trimmed cotyledons were also transferred to a fresh shoot induction medium containing $75 \mathrm{mg} / \mathrm{L}$ kanamycin for another 2 weeks. Afterwards, the cotyledons were transferred onto shoot elongation medium (SEM) containing $75 \mathrm{mg} / \mathrm{L}$ kanamycin every two weeks until enough elongating shoots were obtained. $2-3 \mathrm{~cm}$ long putative transgenic shoots were rooted on rooting medium and transferred into pots containing soil. $\mathrm{T}_{3}$ transgenic soybean seeds were obtained for further analysis.

\subsection{Generation of GmSOG1 Overexpressing Arabidopsis Lines}

The full-length open reading frame of GmSOG1 was amplified from the cDNA of soybean leaves using gene specific primers and cloned into the PCR8 entry vector. After sequencing, the GmSOG1 gene was inserted downstream of the CaMV 35S promoter at the attR1-attR2 sites of the pGWB2 binary vector [22] by means of LR cloning. For Arabidopsis thaliana transformation, Agrobacterium tumefaciens EHA105 cells bearing the pGWB2::GmSOG1 were transformed into Arabidopsis plants via the floral inoculation method [23]. $\mathrm{T}_{0}$ transgenic seeds were harvested and sown on MS media containing $50 \mathrm{mg} / \mathrm{L}$ kanamycin and 20 $\mathrm{mg} / \mathrm{L}$ meropenem. Kanamycin selection of putative seeds was carried out until $\mathrm{T}_{3}$ homozygous lines were obtained. Lines 1,2 and 3 were selected for further analysis.

\subsection{RT-PCR of Transgenic Soybean Plants}

RNAs extracted from wild-type and transgenic soybean plants were converted to cDNA using the PrimeScript RT reagent with gDNA eraser kit (TaKaRa Bio Inc, Japan). Using cDNAs as template the GUS linker sequence in the pANDA35HK vector was amplified according to the following PCR conditions; initial denaturation: $94^{\circ} \mathrm{C}$ for 2 minutes, denaturation: $94^{\circ} \mathrm{C}$, for 10 seconds, annealing: $62^{\circ} \mathrm{C}$ for 10 seconds and extension: $68^{\circ} \mathrm{C}$ for 15 seconds. Amplifications were carried out for 30 cycles with the soybean elongation factor $1 \alpha$ (GmEF1a) as an internal control gene.

\subsection{Real Time PCR}

Quantitative real time PCR was carried out with the KOD SYBR qPCR Mix 
(TOYOBO) on a Step One Plus Real-Time PCR System machine (Applied Biosystems). Triplicates of $20 \mu \mathrm{l}$ reaction mixture were set up for each treatment.

\subsection{In Vitro Drought Stress Analysis of Soybean Plants}

Seeds of T3 transgenic and wild-type soybean plants (8 seeds per line for a single independent experiment) were germinated on MSB5 medium for three days and then transferred onto fresh MSB5 media containing $200 \mathrm{mM}$ mannitol for 10 days. After ten days of growth, parameters such as weight of root mass and length of primary roots of individual plants were measured.

\subsection{Drought Stress Analysis of Transgenic Soybean Plants on Soil}

Seeds of wild-type and $\mathrm{T}_{3}$ transgenic soybean plants were germinated on MSB5 medium for 7 days. Afterwards, 10 plants (for a single independent experiment) of each line were transferred onto soils and grown in a plant growth chamber under $16 / 8$ photoperiod. At 2 weeks, soybean plants were irrigated with $15 \%$ polyethylene glycol (PEG) for 10 days. Subsequently, plants were recovered with water for 3 days and parameters such as rate of survival and plant fresh weights taken. Plant survival rate was computed as the percentage of soybean plants not showing signs of whole plant wilting (plants with less than $90 \%-100 \%$ of leaves not showing signs of wilting).

\subsection{Drought Stress Analysis of Transgenic Arabidopsis Plants}

Seeds of $\mathrm{T}_{3}$ transgenic Arabidopsis plants and wild-type Arabidopsis thaliana (Columbia-0 ecotype, Col-0) were surface-sterilized with $1 \%$ sodium hypochlorite solution for 10 mins, rinsed five times in sterile double-distilled water, plated on $1 / 2 \mathrm{MS}$ medium and vernalized in the dark at $4^{\circ} \mathrm{C}$ for 2 days. The plates were taken out after two days and grown at $22^{\circ} \mathrm{C}$ under $16 \mathrm{~h}$ light $/ 8 \mathrm{~h}$ dark cycles for 12 more days. The seedlings were then transferred to soil and grown under the same conditions until the third week. 3-weeks-old seedlings (10 plants per line for a single independent experiment) were subjected to drought stress by withholding water for 14 days, followed by recovery by watering for 3 days.

\subsection{Rate of Water Loss}

The rate of water loss in detached Arabidopsis leaves was determined according to the methods of [24] [25]. The rate of transpiration of detached Arabidopsis leaves was determined over a 6-hour period. Three sets of readings were taken for the Fresh weight (LWt) of leaves at every $60 \mathrm{~min}$ interval and the relative water loss $(\mathrm{RWL})$ of leaves determined as follows: $\mathrm{RWL}=(\mathrm{FW}-\mathrm{LWt}) / \mathrm{FW} \times 100$, where LWt is the weight of the leaf subjected to desiccation treatment for $t$ hours, and FW is the weight of leaves before desiccation.

\subsection{Lipid Peroxidation and Chlorophyll Content Determination}

Malondialdehyde (MDA) content, a marker of lipid peroxidation, was deter- 
mined in leaves according to the method previously described by Heath and Packer [26]. Leaf samples were ground with liquid nitrogen, suspended in $0.1 \%$ trichloroacetic acid (TCA), vortexed and centrifuged at 10,000 rpm for 5 mins. Next, $1 \mathrm{ml}$ of the supernatant was dispensed into a separate tube and $4 \mathrm{ml}$ of $0.5 \%$ thiobarbituric acid (TBA) added. The mixture was incubated at $95^{\circ} \mathrm{C}$ for 30 mins. After incubation, the reaction was quickly cooled on ice and centrifuged at $5000 \mathrm{rpm}$ for 5 mins to settle debris. Absorbances of the supernatants were measured at 532 and $600 \mathrm{~nm}$. Unspecific turbidity was corrected for by subtracting the value at $600 \mathrm{~nm}$. MDA concentration was determined according to an extinction (ع) of $155 \mathrm{mM}^{-1} \cdot \mathrm{cm}^{-1}$ and expressed as nanomoles per gram of fresh weight of leaf samples used.

The chlorophyll contents of plants were determined according to the method of Zhang et al. [27]. $0.1 \mathrm{~g}$ of leaf tissues were ground to a fine powder with liquid nitrogen and homogenized with $1 \mathrm{ml}$ 100\% DMF. The homogenized samples were centrifuged for 10 mins and the supernatants collected into fresh tubes. The optical densities of supernatants were measured at $664 \mathrm{~nm}$ and $647 \mathrm{~nm}$ and the total chlorophyll content determined according to the formula: [chlorophyll $\mathrm{a}+$ chlorophyll b] $=17.90 \times \mathrm{A} 647+8.08 \times \mathrm{A} 664$.

\subsection{Determination of Ascorbate Peroxidase (APX) and Catalase (CAT) Contents}

The activities of APX and catalase CAT were determined in the supernatants of $0.02 \mathrm{~g}$ of Arabidopsis leaves homogenized with $50 \mathrm{mM}$ ice-cold phosphate buffer ( $\mathrm{pH}$ 7.8) containing $1 \mathrm{mM}$ EDTA. The activity of APX (EC 1.11.1.6) was assayed in accordance with the method of Nagano and Asada [28]. A total of $3 \mathrm{ml} \mathrm{reac-}$ tion mixture containing $50 \mathrm{mM}$ phosphate buffer ( $\mathrm{pH}$ 7.8), $0.1 \mathrm{mM}$ EDTA, 0.5 $\mathrm{mM}$ ascorbate, $0.1 \mathrm{mM} \mathrm{H}_{2} \mathrm{O}_{2}$ and $0.2 \mathrm{ml}$ enzyme extract was set up. The activity of APX was determined spectrophotometrically by measuring the hydrogen peroxide induced oxidation of ascorbate at $290 \mathrm{~nm}$ for 3 mins at 30 seconds intervals. The molar extinction coefficient for ascorbate $2.8 \mathrm{mM}^{-1} \cdot \mathrm{cm}^{-1}$ was used to quantify APX activity. CAT activity was determined following the method outlined by Cakmak and Marschner [29] in $3 \mathrm{ml}$ of reaction solution consisting of $100 \mathrm{mM}$ phosphate buffer (pH7.0), 0.1 mM EDTA, $0.1 \% \mathrm{H}_{2} \mathrm{O}_{2}$ and $0.2 \mathrm{ml}$ enzyme extract. CAT activity was quantified at an extinction enzyme extract co-efficient of $39.4 \mathrm{mM}^{-1} \cdot \mathrm{cm}^{-1}$ after the rate of decrease hydrogen peroxide measured at 240 $\mathrm{nm}$ on a spectrophotometer.

\subsection{Statistical Analysis}

The SPSS software (SPSS Inc., Chicago, IL, USA) was used to analyze the data generated. The Duncan's test was used to separate differences in means between treatments at a probability level of 0.05 . Graphs were generated with Microsoft Excel. All experiments were performed in triplicates. Sequences of primers used in this work are given in Table 1. 
Table 1. List of primers used in this study.

\begin{tabular}{|c|c|}
\hline Primer name & Sequences $\left(5^{\prime}-3^{\prime}\right)$ \\
\hline GmLEAFP & TGGGTAATATTGGGCGAAGGAATG \\
\hline GmLEARP & AATTTCAGGGGTGTGGTTAATGGG \\
\hline GmDREB1FP & AGGAAGAAGAGCAGGTGTTGGATA \\
\hline GmDREB1RP & GGCATCCAAGTCAGCATCTTCATA \\
\hline GmAPX1FP & GTTGTTGAGTGGTGAGAAGGAAGG \\
\hline GmAPX1RP & GCATATTTATCAACGAGAGGGCGG \\
\hline GmCAT1FP & CCTGCATTTTGCCCTGCCATTATT \\
\hline GmCAT1RP & TCCAAGTCTGTGTCTCTGGGTATC \\
\hline GmEF1a5P & ACTGTGCTGTCCTGATTATTGACT \\
\hline GmEF1a3P & GGACCAAAAGTAACAACCATACCA \\
\hline HPTFP & CAGCTTCGATGTAGGAGGGCGTGG \\
\hline HPTRP & AATCCCCGAACATCGCCTCGCTCC \\
\hline GmSOG1RNAiFP & AGGTGAATATGTGGTTTCAAAAAT \\
\hline GmSOG1RNAiRP & GAAAGTAAATTTTAGTGAACCAAG \\
\hline GmSOG1FP & СTCTTCTTCGATACAATGGCTAGG \\
\hline GmSOG1RP & CGCATAACCTGTCCATCCAATCAA \\
\hline Gus linkerFP & CATGAAGATGCGGACTTACG \\
\hline Gus linkerRP & ATCCACGCCGTATTCGG \\
\hline
\end{tabular}

\section{Results}

\subsection{Phylogenetic and Expression Analysis of GmSOG1 in Soybean Tissues}

To understand the evolutionary relationship that exists between GmSOG1 and homologous proteins from other plants, a phylogenetic tree was constructed. The results of phylogenetic analysis revealed that GmSOG1 was closely related with a homologous protein in Gossypium hirsutum but distantly related with a similar protein in Arabidopsis thaliana (Figure 1(A)). Gene expression analysis of GmSOG1 in unstressed, drought stressed, and salt stressed soybean tissues showed the expression of the gene in all tissues tested. GmSOG1 was highly expressed in buds, young leaves and young shoots. Immature soybean pods on the other hand showed the lowest level of expression (Figure 1(B)). 
(A)

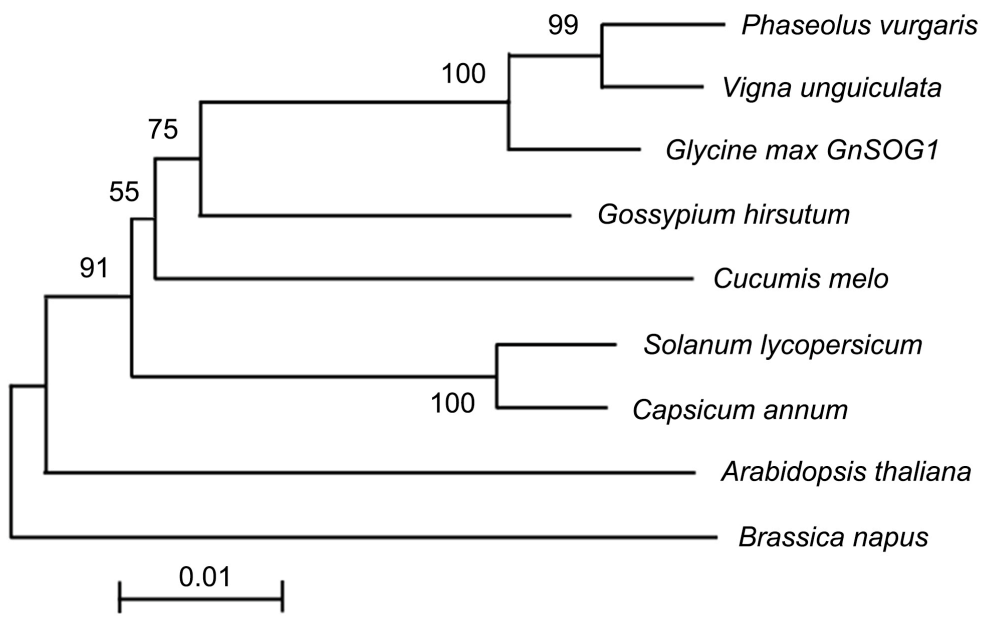

(B)

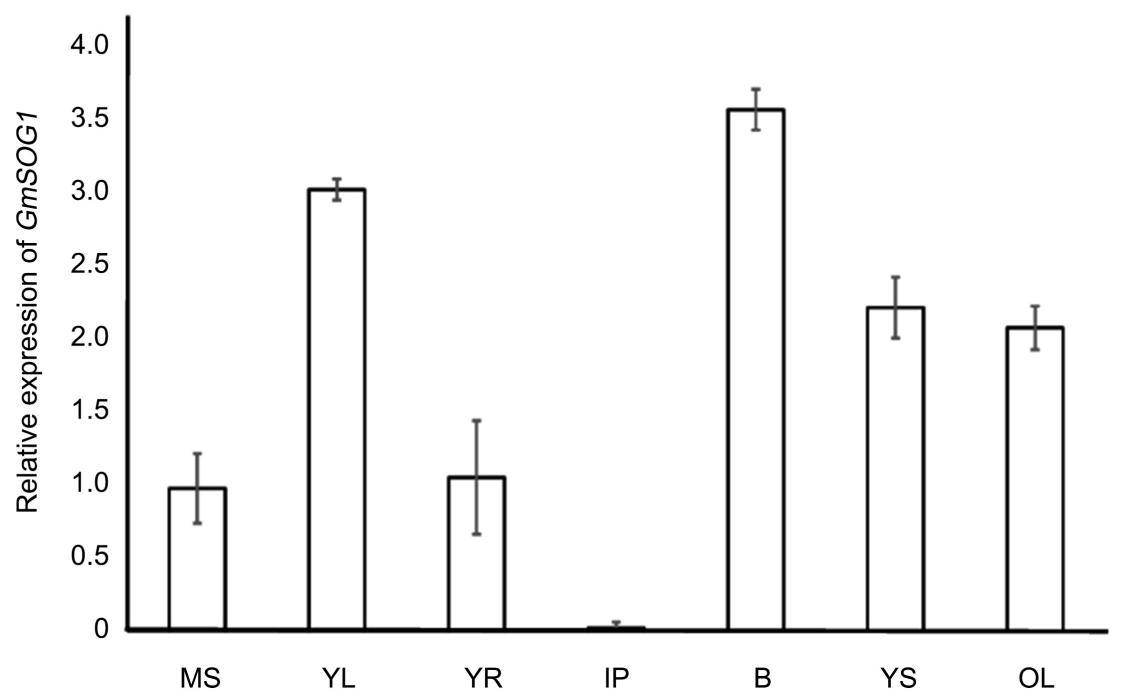

Figure 1. Phylogenetic and expression analysis of GmSOG1. (A) Phylogenetic relationships between GmSOG1 and homologous proteins from Phaseolus vulgaris, Arabidopsis thaliana, Vigna unguiculata, Solanum lycopersicum, Capsicum annuum, Gossypium hirsutum and Brassica napus. (B) Expression analysis of GmSOG1 in tissue samples of drought stressed soybean plants. MS: mature shoots, YL; young leaves, YR: young roots, IP: immature pods, B: buds, YS: young shoots, OL: old leaves. Data are means of triplicates from three independent experiments. Error bars indicate SE.

\subsection{Generation of GmSOG1 Transgenic Soybean Lines}

We generated six putative transgenic lines. Out of this number, only two lines could produce viable seeds. These lines were denoted as R1 and R2. qRT-PCR analysis confirmed the successful knockdown of the GmSOG1 gene in transgenic soybean lines. The GmSOG1 gene was found to be highly downregulated in R1 plants than R2 plants. On average, Knockdown transgenic lines showed about a 1.92-fold decrease in GmSOG1 expression relative to the wild-type (Figure $2(\mathrm{~A})$ ). Furthermore, transgenic plants were shown to be RT-PCR positive. The GUS-linker sequence of the pANDA35HK vector was successfully amplified from cDNAs isolated from transgenic soybean lines (Figure 2(B)). 


\subsection{In Vitro Drought Stress Analysis of Soybean Plants}

The drought tolerance capacities of soybean plants were evaluated based on root length and root fresh weight measurements of wild-type and transgenic plants (8 plants per line) subjected to drought stress on Murashige and Skoog medium plus B5 vitamins (MSB5) media containing $200 \mathrm{mM}$ mannitol. After 10 days of droughts stress treatment, root lengths and root fresh weights were significantly higher in wild type soybean plants compared with RNAi transgenic lines (Figure 2(C), Figure 2(D)).

\subsection{Drought Stress Assays of Soybean Plants on Soil}

The survival rates of wild-type soybean plants subjected to PEG-induced drought stress were significantly higher than transgenic lines. More than $70 \%$ of the tested wild-type plants survived after recovery whereas less than $10 \%$ of the two transgenic lines tested showed signs of recovery (Figure 3(A), Figure 3(B)). The fresh weights of wild-type soybean plants after drought stress treatment were also significantly higher than RNAi transgenic lines (Figure 3(C)).

(A)

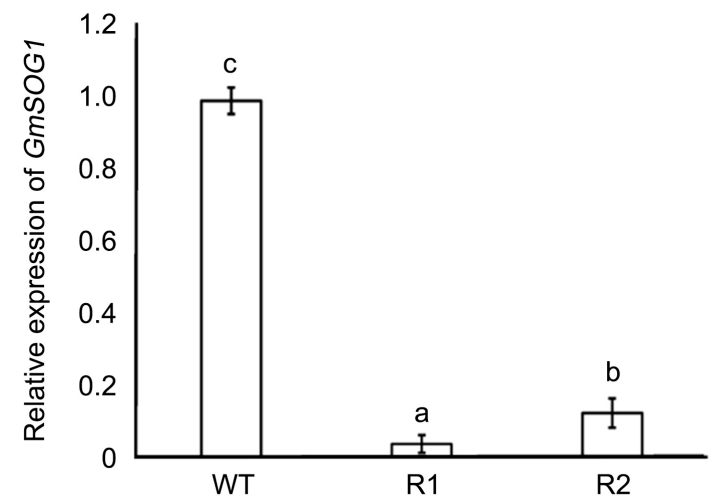

(B)

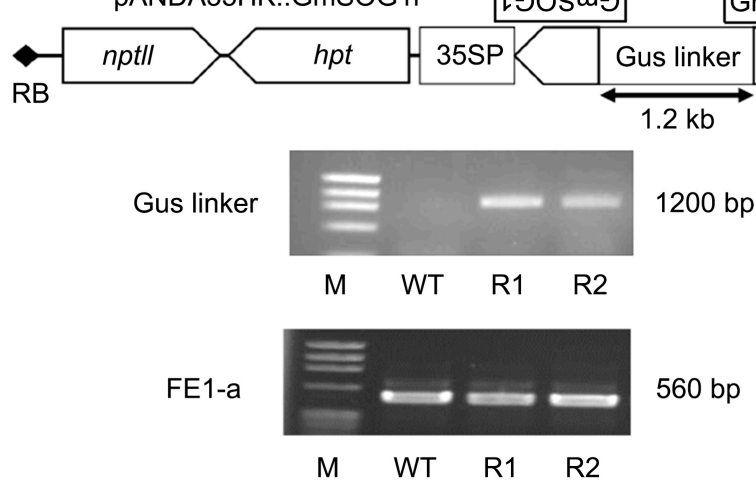

(C)

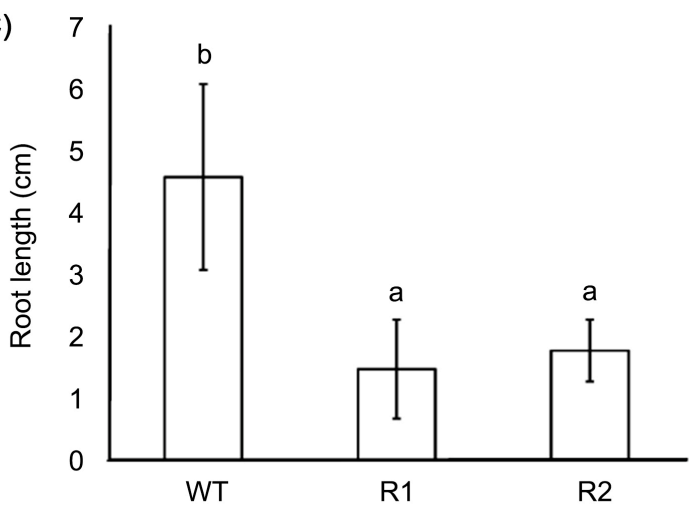

(D)

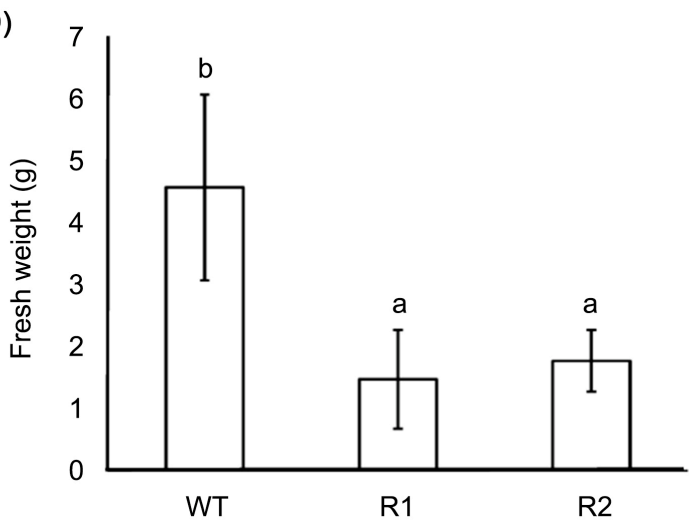

Figure 2. Molecular and Physiological analysis of soybean lines. (A) Relative expression analysis of GmSOG1 in wild-type and RNAi-transgenic soybean lines by qRT-PCR. (B) RT-PCR analysis of the Gus linker sequence (1200 bp) of introduced RNAi construct (pANDA35HK::GmSOG1i) and EF-1a (560 bp as internal control) in soybean lines. M: marker, nptII: neomycin phosphotransferase II gene, $h p t$. hygromycin phosphotransferase gene, 35SP: CaMV $35 \mathrm{~S}$ promoter, nos T: nopaline synthase gene terminator, RB: right border, LB: left border. (C) Root length analysis of soybean plants. (D) Root fresh weight analysis of soybean plants WT: wild-type, R1: RNAi-transgenic line 1, R2: RNAi-transgenic line 2. Different letters represent significant difference $(\mathrm{p}<0.05)$ according to Duncan's test. 
(A)

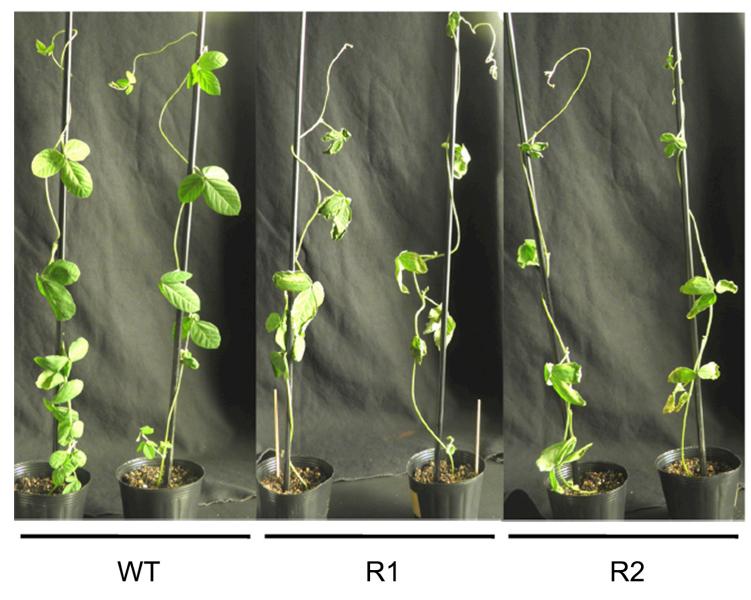

(B)

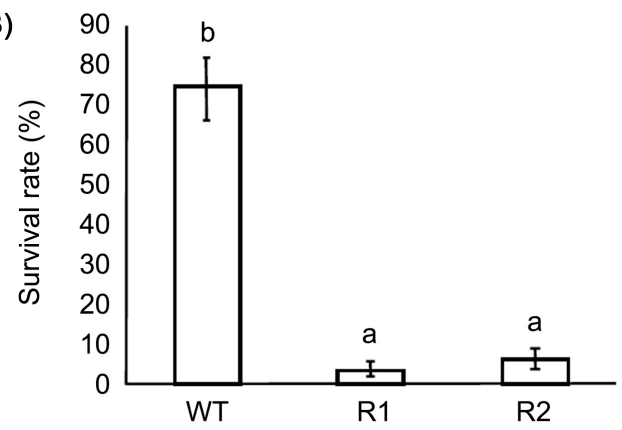

(C)

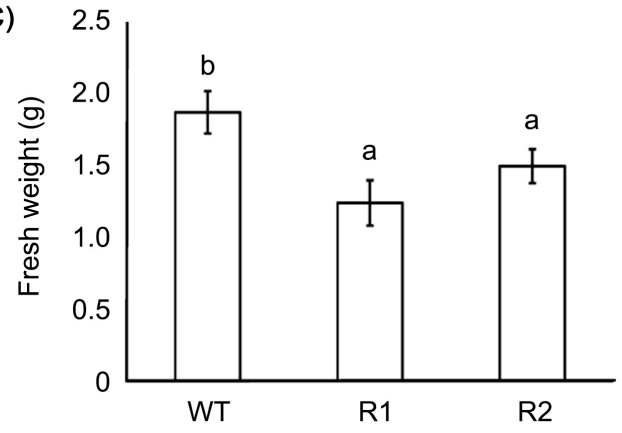

Figure 3. Drought tolerance assays of soybean lines. (A) Phenotypes of soybean lines treated with 15\% PEG. (B) Survival rates (\%) of drought stressed soybean lines. (C) Fresh weight (g) analysis of soybean lines. WT: wild-type, R1: RNAi-transgenic line 1, R2: RNAi-transgenic line 2. Data are means SD of triplicates from three independent experiments. Error bars denote SD. Bars with different letters represent significant difference $(\mathrm{p}<0.05)$ according to Duncan's test.

\subsection{Relative Expression of Transcription Factors}

The relative expression levels of two transcription factors: the soybean dehydration responsive element binding protein 1 gene (GmDREB1) and the soybean late embryogenesis abundant protein 5 gene (GmLEA-5) were investigated in leaves of unstressed and drought stressed wild-type and GmSOG1 knockdown soybean lines. The transcript levels of the two transcription factors tested were low and showed no significant differences between wild-type and knockdown lines in unstressed leaf samples. The rates of expression of both genes were however, upregulated in response to drought stress in all lines tested. Expression levels of the genes in drought stressed tissues were significantly higher in the wild-type line than transgenic lines (Figure 4(A), Figure 4(B)).

\subsection{Relative Expression of Anti-Oxidation Related Genes}

The relative expression levels of anti-oxidation related genes such as GmAPX and $G m C A T$ were studied in wild-type and knockdown transgenic soybean lines before and after drought stress application. The differences in the transcript levels of the two genes were minute in unstressed wild-type and transgenic lines. However, under drought stress, the wild-type line showed significantly higher transcription levels of both GmAPX and GmCAT relative to transgenic lines (Figure 4(C), Figure 4(D)). After drought stress application, on average, wild type plants accumulated approximately 10 times more $G m A P X$ and 12 times more GmCAT1 transcripts than transgenic plants (Figure 4(C), Figure 4(D)). 


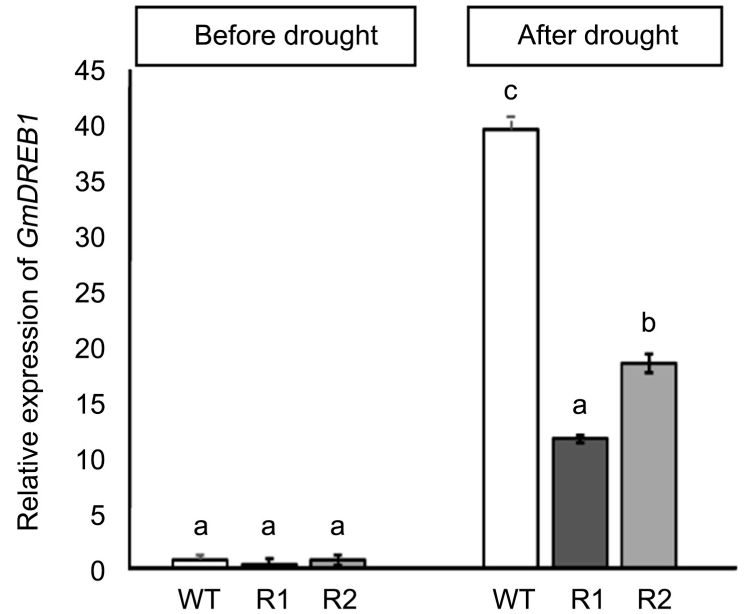

(A)

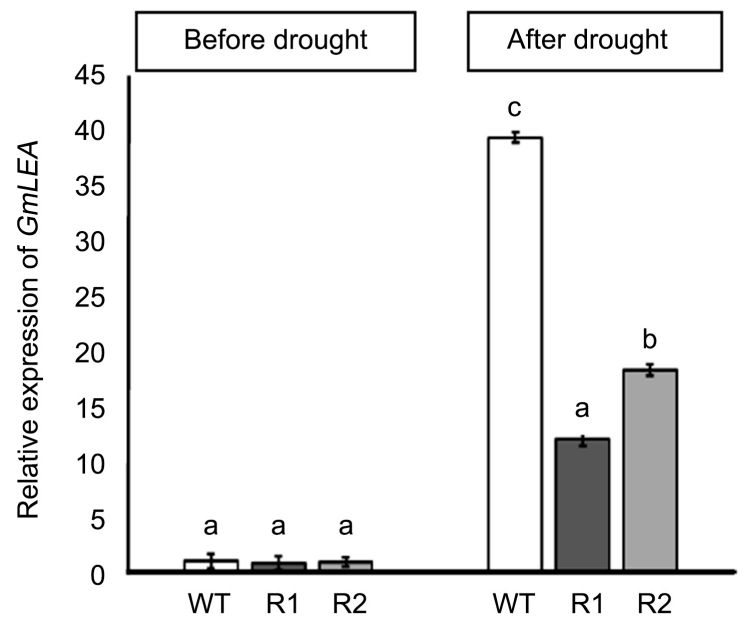

(B)

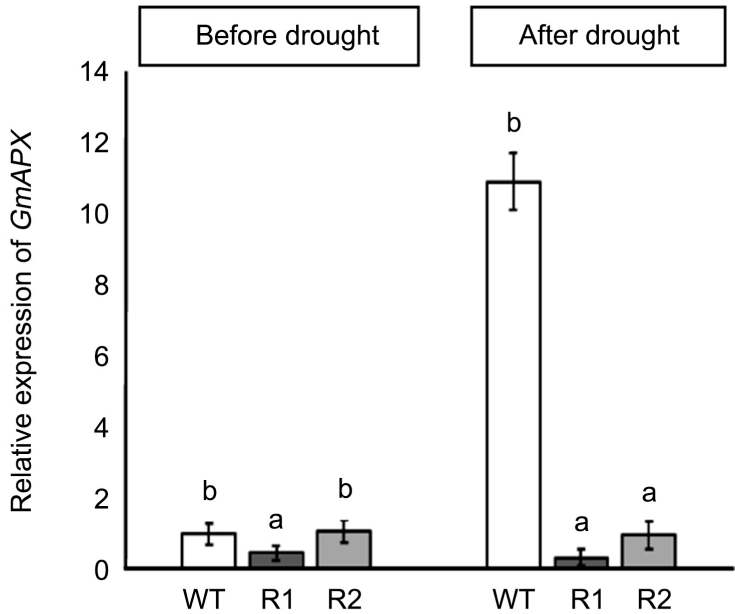

(C)

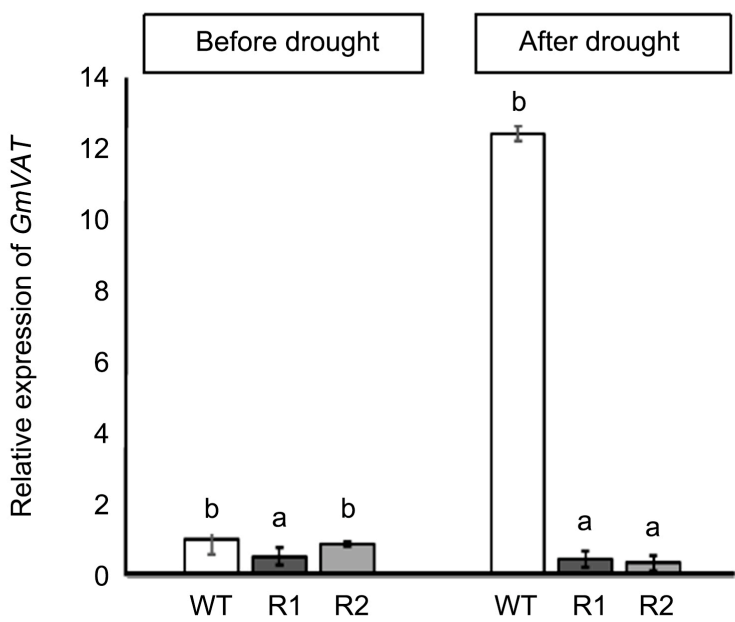

(D)

Figure 4. Expression analysis of transcription factors and anti-oxidation related genes. (A) qRT-PCR analysis of GmDREB1 in soybean plants. (B) qRT-PCR analysis of $G m L E A$ in soybean plants (C) qRT-PCR analysis of the $G m A P X$ in soybean plants. (D) qRT-PCR analysis of GmCAT in soybean plants. WT: wild-type. R1: RNAi-transgenic line 1, R2: RNAi-transgenic line 2. Data are means of triplicates from three independent experiments. Error bars indicate SD. Bars with different letters represent significant difference $(\mathrm{p}<0.05)$ according to Duncan's test.

\subsection{Relative MDA and Chlorophyll Contents}

The relative amount of MDA was found to be significantly higher in knockdown transgenic soybean lines than in wild-type plants under both non-stressed and drought stressed conditions, indicating a higher level of lipid peroxidation in transgenic lines compared with wild-type plants. Averagely, unstressed wild-type soybean plants accumulated 2.31 times the amount of MDA in unstressed transgenic plants. Drought stressed wild-type soybean plants on the other hand accumulated 2.24 times the amount of MDA in transgenic plants under the same condition (Figure 5(A)). The chlorophyll contents of wild-type and transgenic plants were also significantly different under both non-stressed and drought stressed conditions. Transgenic plants accumulated significantly lesser amounts 
of chlorophyll compared to wild-type plants. On average, drought stressed wild-type soybean plants accumulated 1.4 times the amount of chlorophyll in transgenic plants under the same condition (Figure $5(B)$ ).

\subsection{Drought Stress Analysis of Transgenic Arabidopsis Plants}

3-weeks-old Arabidopsis seedlings were subjected to drought stress by with-holding water for 14 days. At the end of the drought stress application period, plants were recovered with water for 3 days. After drought stress application and recovery, all transgenic plants tested survived whereas none of the wild-type plants tested showed signs of recovery (Figure 6(A), Figure 6(B)). The rate of water loss in detached transgenic Arabidopsis leaves was also investigated over a period of six hours. Our results showed that leaves of wild-type plants had lost a significantly higher amount of water compared with transgenic plants. The wild-type line lost about $65 \%$ of its water content compared with a water loss rate of $25 \%, 34 \%$ and $39 \%$ for line 3, line 1 and line 2 transgenic Arabidopsis plants respectively (Figure 6(C)).

\subsection{Enzymatic Activities of APX and CAT in Arabidopsis Plants}

The activities of APX and CAT were assayed in Arabidopsis plants prior and after drought stress treatment. The activities of the two enzymes tested were not significantly different in unstressed plants except in line 3. However, both APX and CAT showed significantly higher activities in drought stressed transgenic Arabidopsis lines compared to wild-type plants. On average, Transgenic lines accumulated 0.68 times more APX and 1.18 times more CAT than wild-type plants (Figure 7(A), Figure 7(B)).

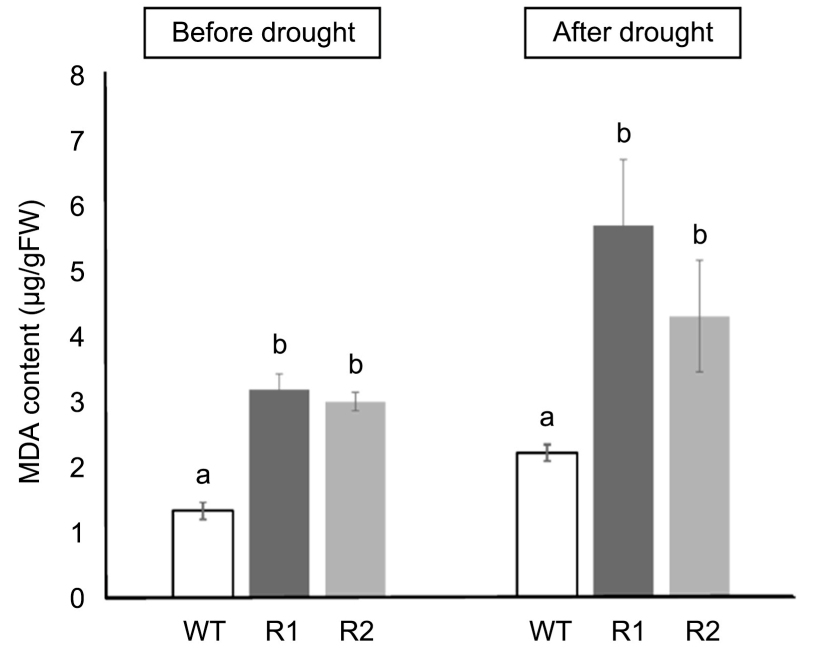

(A)

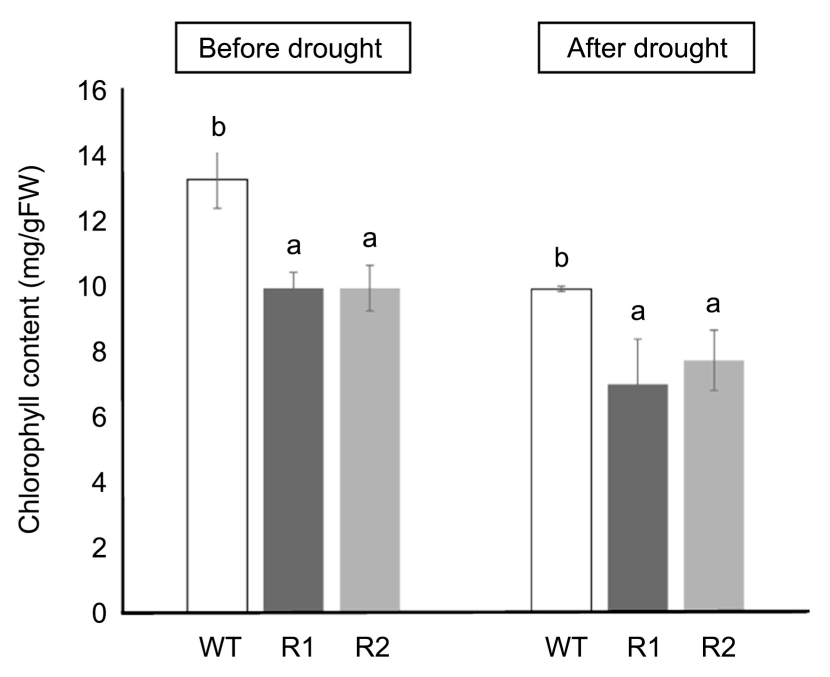

(B)

Figure 5. Biochemical analysis of soybean lines pre and post drought stress treatment. (a) MDA content ( $\mu \mathrm{g} / \mathrm{gFW}$ ) of soybean lines before and after drought stress application (b) Chlorophyll content (mg/gFW) of soybean lines. WT: wild-type, R1: RNAi-transgenic line 1, R2: RNAi-transgenic line 2. Data are means of triplicates from three independent experiments. Error bars represent SD. Different letters represent significant difference $(\mathrm{p}<0.05)$ according to Duncan's test. 

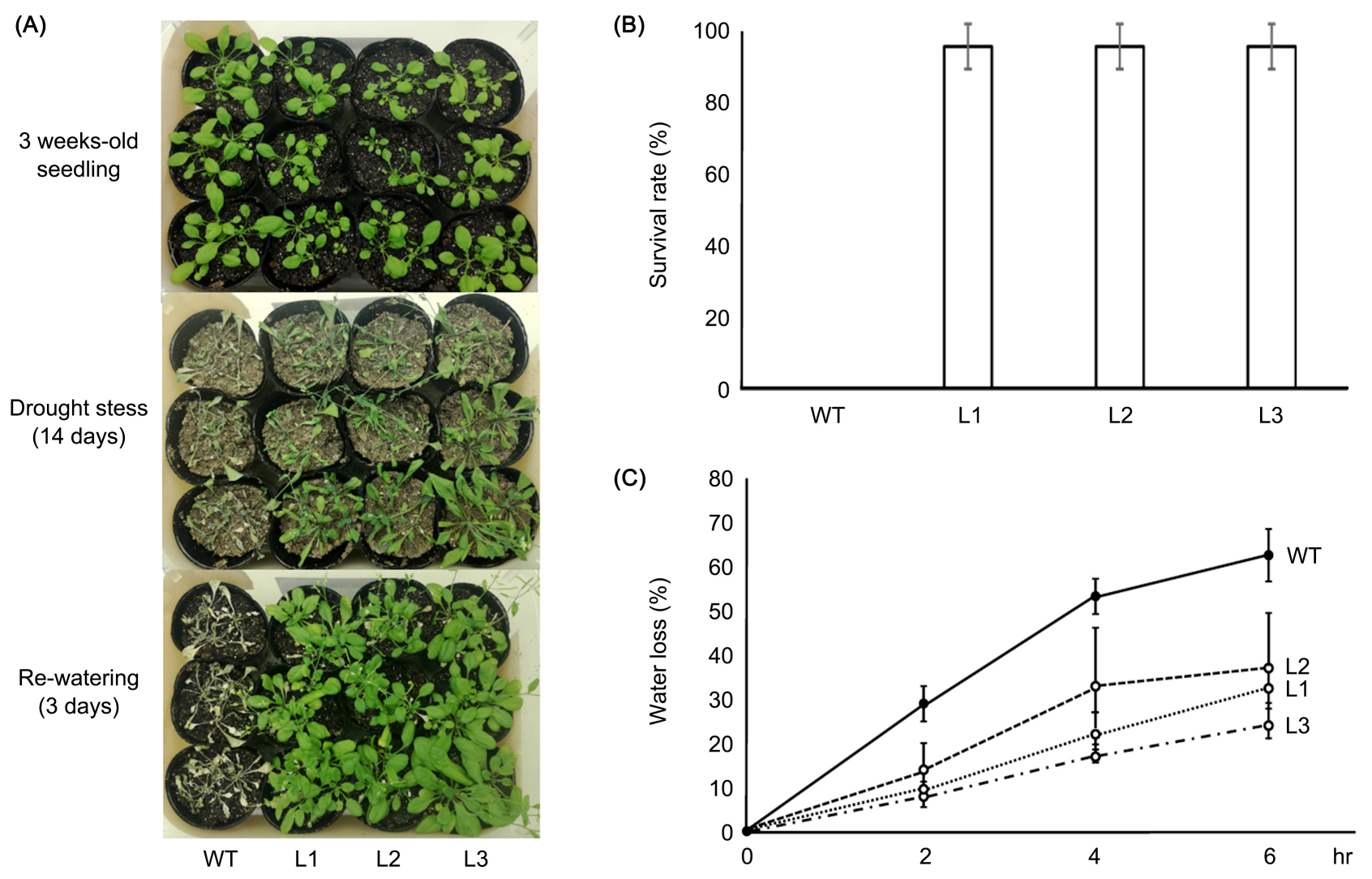

Figure 6. Responses of Arabidopsis lines to drought. (A) Phenotypes of 3-weeks-old Arabidopsis seedlings subjected to drought stress (14 days) followed by re-watering (3 days). (B) Survival rates (\%) of Arabidopsis plants after drought stress treatment. C: Percentage rate of water loss in Arabidopsis lines. WT: wild type, L1: transgenic line 1, L2: transgenic line 2, L3: transgenic line 3. Data are means of triplicates from three independent experiments. Error bars represent SD. Bars with different letters represent significant difference $(\mathrm{p}<0.05)$ according to Duncan's test.

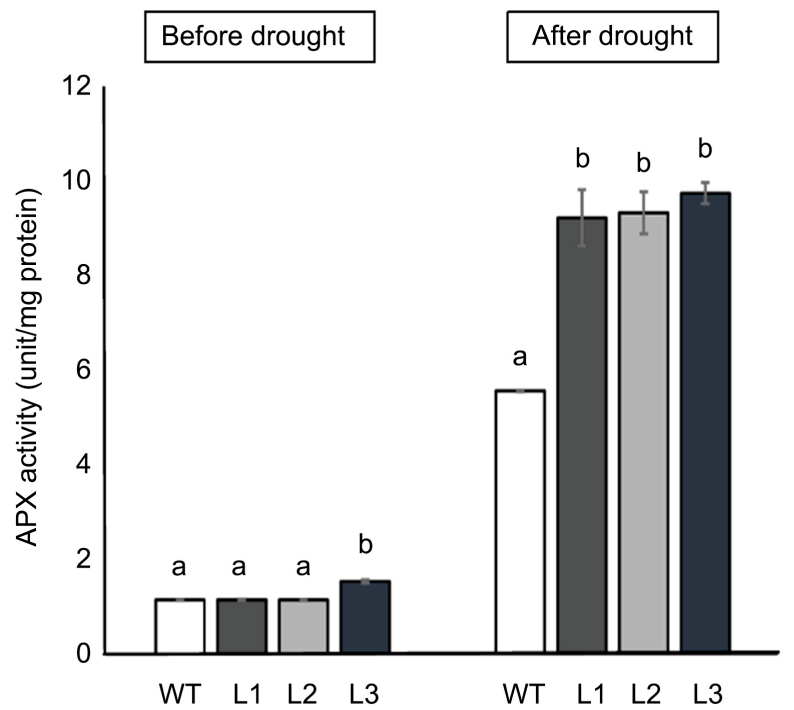

(A)

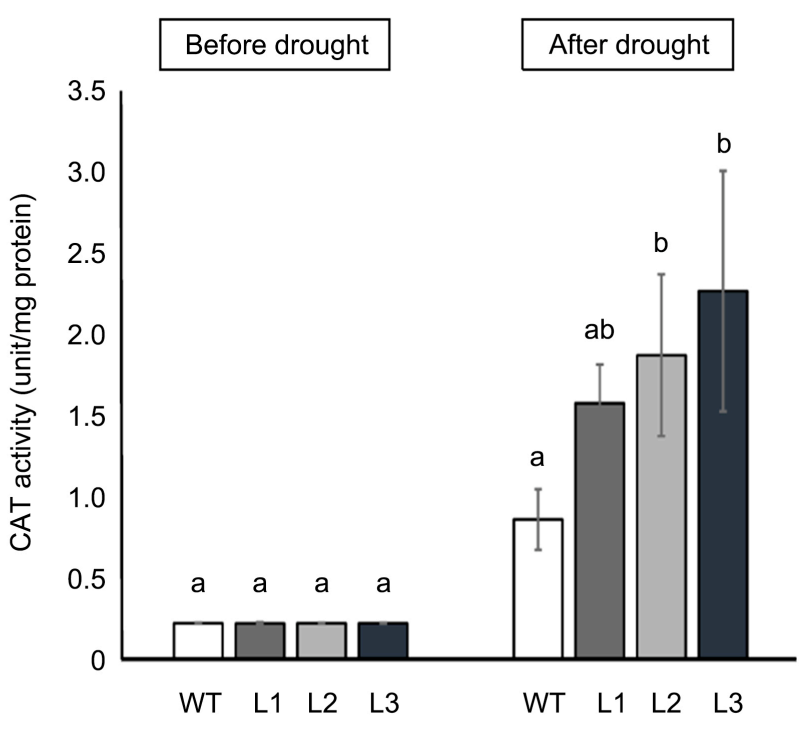

(B)

Figure 7. Enzymatic activities of APX and CAT in Arabidopsis lines. (A) Enzymatic activity of APX in unstressed and drought stressed Arabidopsis lines. (B) Enzymatic activity of CAT in unstressed and drought stressed Arabidopsis. Data are means of triplicates from three independent experiments. Error bars represent SD. 


\section{Discussion}

NAC genes are a major group of transcription factors that play major roles in plant root development and stress responses. This family of transcription factors contains several valuable candidate genes that can be used for plant drought stress improvement [30]. In this report, we studied the roles of GmSOG1 in drought stress tolerance.

Soybean plants when exposed to drought stress exhibit morphological changes in their growth [31]. One vital part of the soybean plant that shows changes in its morphology in response to drought stress is its root system [32] thus root traits have been proposed as useful drought tolerance indicators in soybean plants [33] [34]. A positive relationship has been found to exist between soybean root traits such as root weight and drought stress tolerance [31]. In this study, wild-type soybean plants recorded significantly higher root lengths and root fresh weights compared with GmSOG1 knockdown transgenic lines (Figure 2(C), Figure 2(D)). Evidence support the fact that deep penetrating roots with larger lateral root systems are advantageous under drought conditions [35]. Research has shown that roots with such architectures possess a greater total surface area and hence have the capacity to ensure maximum water and nutrient uptake to sustain photosynthesis under stress conditions [35].

Plant weight parameters have also been identified as useful markers for soybean drought tolerance [31]. The weight of plants after drought stress treatment can give an indication as to the extent to which the plants could maintain metabolism under such conditions. Our results showed that wild-type soybean plants had significantly higher fresh weights after drought stress recovery compared with knockdown transgenic lines (Figure 3(C)). The survival rates of wild-type plants were also significantly higher than knockdown transgenic lines (Figure 3(A), Figure 3(B)). A report on the functional study of the soybean delta-1-pyrroline-5-carboxylate synthase gene (GmP5CS) revealed higher rates of survival in drought stressed wild-type plants compared with P5CS knocked-down transgenic soybean lines [36]. In a confirmatory experiment involving GmSOG1 overexpressing Arabidopsis plants, all transgenic lines tested showed signs of recovery after recovery from drought stress whereas none of the wild-type plants could survive (Figure 6(A), Figure 6(B)).

Transcription factors play vital roles in plant stress defense by regulating the expression of other genes. We investigated the effects the downregulation of GmSOG1 would have on two known drought responsive genes: the GmLEA5 and $G m D R E B 1$ genes. Our results showed that the downregulation of GmSOG1 expression in transgenic lines resulted in a corresponding downregulation in the expression levels of the two genes tested (Figure 4(A), Figure 4(B)). In line with this result, it has been reported that the AtSOG1 transcription factor regulates the expression of certain abiotic stress responsive genes [37]. Another way plants deal with the deleterious effects of stress factors is by making changes in the expression profiles of antioxidation related genes [38]. Antioxidants play integral 
roles in both redox systems and DDR pathways to counteract the harmful effects of genotoxic stress in plants [39]. In this research, antioxidation related genes such as GmAPX and GmCAT were all downregulated in knockdown transgenic soybean plants relative to wild-type plants (Figure 4(C), Figure 4(D)). Furthermore, the activities of APX and CAT were greatly enhanced in GmSOG1 overexpressing transgenic Arabidopsis plants (Figure 7(A), Figure 7(B)).

Plants produce reactive oxygen species as a result of normal metabolic activities and in response to environmental stresses. The accumulation of these compounds in plant cells causes lipid peroxidation which adversely impacts the chlorophyll content of plants [40]. In this study, GmSOG1 knockdown transgenic lines accumulated significantly higher amounts of MDA than wild-type plants under both normal and drought stressed conditions (Figure 5(A)). The accumulation of ROS due to normal cellular activities has been identified as a major cause of DNA damage in plants [14]. The downregulation of GmSOG1, a major plant DNA damage responsive gene, in transgenic lines possibly interfered with the mechanisms of ROS scavenging hence the higher accumulation of MDA in transgenic plants. A significantly higher amount of chlorophyll was also observed in wild-type soybean plants relative to transgenic lines (Figure 5(B)). The chloroplast of plants has been identified as a major site for the production of reactive oxygen species (ROS) [41]. The buildup of ROS in the photosynthetic machinery of plants as a result of stresses, may lead to chlorophyll degradation [40] [42] [43]. Transgenic soybean lines therefore had lower amounts of chlorophyll under drought stress as they may have lacked a protective mechanism against ROS induced chlorophyll degradation.

\section{Conclusion}

We have shown that there may exist an interplay between the DNA damage response (DDR) pathway and abiotic stress response in plants. The GmSOG1 gene, a chief regulatory gene of the DDR pathway was found to play roles in drought tolerance. The suppression of GmSOG1 expression in soybean plants led to a compromised ROS scavenging system under drought conditions. The SOG1 gene is a potentially useful candidate gene for plant stress engineering hence the need to identify and functionally characterize homologous genes in other plant species.

\section{Acknowledgements}

The authors express their sincere gratitude to Dr. Hiroyuki Tsuji of Kihara Institute of Biological Research, Yokohama City University, Japan for providing us with the pANDA35HK RNAi binary vector. The authors would also like to thank the Japanese Ministry of Education, Culture, Sports, Science and Technology (MEXT) for the award of scholarship to S. A. Poku.

\section{Conflicts of Interest}

The authors declare no conflict of interest regarding publication of this manuscript. 


\section{References}

[1] Goldsmith, P.D. (2008) Economics of Soybean Production, Marketing, and Utilization. In: Johnson, L.P., White, P.A. and Galloway, R., Eds., Soybeans. Chemistry, Production, Processing and Utilization, AOCS Press, Urbana, 117-150. https://doi.org/10.1016/B978-1-893997-64-6.50008-1

[2] Sakai, T. and Kogiso, M. (2008) Soy Isoflavones and Immunity. Journal of Medical Investigation, 55, 167-173. https://doi.org/10.2152/jmi.55.167

[3] Friedman, M. and Brandon, D.L. (2001) Nutritional and Health Benefits of Soy Proteins. Journal of Agriculture and Food Chemistry, 49, 1069-1086. https://doi.org/10.1021/jf0009246

[4] Le, D.T., Nishiyama, R., Watanabe, Y., Tanaka, M., Seki, M., Ham, L., Yamaguchi-Shinozaki, K. Shinozaki, K. and Lam, T. (2012) Differential Gene Expression in Soybean Leaf Tissues at Late Developmental Stages under Drought Stress Revealed by Genome-Wide Transcriptome Analysis. PLoS ONE, 7, e49522. https://doi.org/10.1371/journal.pone.0049522

[5] Hirt, H. and Shinozaki, K. (2004) Plant Responses to Abiotic Stress. Springer, Berlin. https://doi.org/10.1007/b84369

[6] Sinclair, T. and Serraj, R. (1995) Legume Nitrogen-Fixation and Drought. Nature, 378, 344. https://doi.org/10.1038/378344a0

[7] Purcell, L.C. and King, C.A. (1996) Drought and Nitrogen Source Effects on Nitrogen Nutrition, Seed Growth, and Yield in Soybean. Journal of Plant Nutrition, 19, 969-993. https://doi.org/10.1080/01904169609365173

[8] Chen, M., Wang, Q.Y., Cheng, X.G., Xu, Z.S., Li, L.C., Ye, X.G., Xia, L.Q. and Ma, Y.Z. (2007) GmDREB2, a Soybean DRE-Binding Transcription Factor, Conferred Drought and High-Salt Tolerance in Transgenic Plants. Biochemical and Biophysical Research Communications, 353, 299-305. https://doi.org/10.1016/j.bbrc.2006.12.027

[9] Zhang, G., Chen, M., Li, L., Xu, Z., Chen, X., Guo, J. and Ma, Y. (2009) Overexpression of the Soybean GmERF3 Gene, an AP2/ERF Type Transcription Factor for Increased Tolerances to Salt, Drought, and Diseases in Transgenic Tobacco. Journal of Experimental Botany, 60, 3781-3796. https://doi.org/10.1093/jxb/erp214

[10] Gao, S.Q., Chen, M., Xu, Z.S., Zhao, C.P., Li, L., Xu, H.J., Tang, Y.M., Zhao, X. and Ma, Y.Z. (2011) The Soybean GmbZIP1 Transcription Factor Enhances Multiple Abiotic Stress Tolerances in Transgenic Plants. Plant Molecular Biology, 75, 537-553. https://doi.org/10.1007/s11103-011-9738-4

[11] Baxter, A., Mittler, R. and Suzuki, N. (2013) ROS as Key Players in Plant Stress Signaling. Journal of Experimental Botany, 65, 1129-1240. https://doi.org/10.1093/jxb/ert375

[12] Foyer, C.H. and Shigeoka, S. (2011) Understanding Oxidative Stress and Antioxidant Functions to Enhance Photosynthesis. Plant Physiology, 155, 93-100. https://doi.org/10.1104/pp.110.166181

[13] Sancar, A., Lindsey-Boltz, L.A., Unsal-Kaçmaz, K. and Linn, S. (2004) Molecular Mechanisms of Mammalian DNA Repair and the DNA Damage Checkpoints. Annual Review of Biochemistry, 73, 39-85. https://doi.org/10.1146/annurev.biochem.73.011303.073723

[14] Nisa, M.U., Huang, Y., Benhamed, M. and Raynaud, C. (2019) The Plant DNA Damage Response: Signaling Pathways Leading to Growth Inhibition and Putative Role in Response to Stress Conditions. Frontiers in Plant Science, 10, 653. 
https://doi.org/10.3389/fpls.2019.00653

[15] Yoshiyama, K.O., Conklin, P.A., Huefner, N.D. and Britt, A.B. (2009) Suppressor of Gamma Response 1 (SOG1) Encodes a Putative Transcription Factor Governing Multiple Responses to DNA Damage. Proceedings of the National Academy of Sciences, USA, 106, 12843-12848. https://doi.org/10.1073/pnas.0810304106

[16] Yoshiyama, K.O. and Kimura, S. (2018) Ser-Gln Sites of SOG1 Are Rapidly Hyperphosphorylated in Response to DNA Double-Strand Breaks. Plant Signaling \& Behavior, 13, e1477904. https://doi.org/10.1080/15592324.2018.1477904

[17] Adachi, S., Minamisawa, K., Okushima, Y., Inagaki, S., Yoshiyama, K., Kondou, Y., Kaminuma, E., Kawashima, M., Toyoda, T., Matsui, M., Kurihara, D., Matsunaga, S. and Umeda, M. (2011) Programmed Induction of Endoreduplication by DNA Double-Strand Breaks in Arabidopsis. Proceedings of the National Academy of Sciences, USA, 108, 10004-10009. https://doi.org/10.1073/pnas.1103584108

[18] Furukawa, T., Curtis, M.J., Tominey, C.M., Duong, Y.H., Wilcox, B.W., Aggoune, D., Hays, J.B. and Britt, A.B. (2010) A Shared DNA-Damage-Response Pathway for Induction of Stem-Cell Death by UVB and by Gamma Irradiation. DNA Repair, 9, 940-948. https://doi.org/10.1016/j.dnarep.2010.06.006

[19] Larkin, M.A., Blackshields, G., Brown, N.P., Chenna, R., McGettigan, P.A., McWilliam, H., Valentin, F., Wallace, I.M., Wilm, A., Lopez, R., Thompson, J.D., Gibson, T.J. and Higgins, D.G. (2007) Clustal W and Clustal X Version 2.0. Bioinformatics, 23, 2947-2948. https://doi.org/10.1093/bioinformatics/btm404

[20] Tamura, K., Dudley, J., Nei, M. and Kumar, S. (2007) MEGA4: Molecular Evolutionary Genetics Analysis (MEGA) Software Version 4.0. Molecular Biology and Evolution, 24, 1596-1599. https://doi.org/10.1093/molbev/msm092

[21] Hinchee, M.A.W., Connor-Ward, D.V., Newell, C.A., McDonnell, R.E., Sato, S.J., Gasser, C.S., Fischhoff, D.A., Re, D.B., Fraley, R.T. and Horsch, R.B. (1988) Production of Transgenic Soybean Plants Using Agrobacterium-Mediated DNA Transfer. Bio-Technology, 6, 915-922. https://doi.org/10.1038/nbt0888-915

[22] Nakagawa, T., Kurose, T., Hino, T., Tanaka, K., Kawamukai, M., Niwa, Y., Toyooka, K., Matsuoka, K., Jinbo, T. and Kimura, T. (2007) Development of Series of Gateway Binary Vectors, pGWBs, for Plant Transformation. Journal of Bioscience and Bioengineering, 104, 34-41. https://doi.org/10.1263/jbb.104.34

[23] Narusaka, M., Shiraishi, T., Iwabuchi, M. and Narusaka, Y. (2010) The Floral Inoculating Protocol: A Simplified Arabidopsis thaliana Transformation Method Modified from Floral Dipping. Plant Biotechnology, 27, 349-351. https://doi.org/10.5511/plantbiotechnology.27.349

[24] Huang, X.S., Luo, T., Fu, X.Z., Fan, Q.J. and Liu, J.H. (2011) Cloning and Molecular Characterization of a Mitogen-Activated Protein Kinase Gene from Poncirus trifoliata Whose Ectopic Expression Confers Dehydration/Drought Tolerance in Transgenic Tobacco. Journal of Experimental Botany, 62, 5191-5206. https://doi.org/10.1093/jxb/err229

[25] Wang, B.Q., Zhang, Q.F., Liu, J.H. and Li, G.H. (2011) Overexpression of PtADC Confers Enhanced Dehydration and Drought Tolerance in Transgenic Tobacco and Tomato: Effect on ROS Elimination. Biochemical and Biophysical Research Communications, 413, 10-16. https://doi.org/10.1016/j.bbrc.2011.08.015

[26] Heath, R.L. and Packer, L. (1968) Photoperoxidation in Isolated Chloroplasts I. Kinetic and Stoichiometry of Fatty Acid Peroxidation. Archives of Biochemistry and Biophysics, 125, 189-198. https://doi.org/10.1016/0003-9861(68)90654-1

[27] Zhang, Z. and Huang, R. (2013) Analysis of Malondialdehyde, Chlorophyll Proline, 
Soluble Sugar, and Glutathione Content in Arabidopsis Seedling. Bio-Protocol, 3, e817. https://doi.org/10.21769/BioProtoc.817

[28] Nagano, Y. and Asada, K. (1981) Hydrogen Peroxide Is Scavenged by Ascorbate-Specific Peroxidase in Spinach Chloroplasts. Plant Cell Physiology, 22, 867-880.

[29] Cakmak, I. and Marschner, H. (1992) Magnesium Deficiency and High Light Intensity on Enhance Activities of Superoxide Dismutase, Peroxidase and Glutathione Reductase in Bean Leaves. Plant Physiology, 98, 1222-1227. https://doi.org/10.1104/pp.98.4.1222

[30] Hu, H., Dai, M., Yao, J., Xiao, B., Li, X., Zhang, Q. and Xiong, L. (2006) Overexpressing a NAM, ATAF, and CUC (NAC) Transcription Factor Enhances Drought Resistance and Salt Tolerance in Rice. Proceedings of the National Academy of Sciences, USA, 103, 12987-12992. https://doi.org/10.1073/pnas.0604882103

[31] Liu, F., Anderson, M.N., Jacobson, S.E. and Jensen, C.R. (2005a) Stomatal Control and Water Use Efficiency of Soybean (Glycine max L. Merr.) during Progressive Soil Drying. Environmental and Experimental Botany, 54, 33-40.

https://doi.org/10.1016/j.envexpbot.2004.05.002

[32] Benjamin, J.G. and Nielsen, D.C. (2006) Water Deficit Effects on Root Distribution of Soybean, Field Pea and Chickpea. Field Crops Research, 97, 248-253. https://doi.org/10.1016/j.fcr.2005.10.005

[33] Liu, Y., Gai, J.Y., Lu, H.N., Wang, Y.J. and Chen, S.Y. (2005) Identification of Drought Tolerant Germplasm and Inheritance and QTL Mapping of Related Root Traits in Soybean (Glycine max (L.) Merr.). Acta Genetica Sinica, 32, 855-863.

[34] Read, D.J. and Bartlett, E.M. (1972) The Physiology of Drought Resistance in Soybean Plant (Glycine max): The Relationship between Drought Resistance and Growth. Journal of Applied Ecology, 9, 487-489. https://doi.org/10.2307/2402447

[35] Lopes, M.S., Araus, J.L., Van Heerden, P.D.R. and Foyer, C.H. (2011) Enhancing Drought Tolerance in C4 Crops. Journal of Experimental Botany, 62, 3135-3153. https://doi.org/10.1093/jxb/err105

[36] De Ronde, J.A., Spreeth, M.H. and Cress, W.A. (2000) Effect of Antisense L- $\Delta 1$-Pyrroline-5-Carboxylate Reductase Transgenic Soybean Plants Subjected to Osmotic and Drought Stress. Plant Growth Regulation, 32, 13-26. https://doi.org/10.1023/A:1006338911617

[37] Ogita, N., Okushima, Y., Tokizawa, M., Yamamoto, Y.Y., Tanaka, M., Seki, M., Makita, Y., Matsui, M., Okamoto-Yoshiyama, K., Sakamoto, T., Kurata, T., Hiruma, K., Saijo, Y., Takahashi, N. and Umeda, M. (2018) Identifying the Target Genes of Suppressor of Gamma Response 1, a Master Transcription Factor Controlling DNA Damage Response in Arabidopsis. The Plant Journal, 94, 439-453. https://doi.org/10.1111/tpj.13866

[38] Cushman, J.C. and Bohnert, H.J. (2000) Genomic Approaches to Plant Stress Tolerance. Current Opinion Plant Biology, 31, 117-124. https://doi.org/10.1016/S1369-5266(99)00052-7

[39] Foyer, C.H. and Noctor, G. (2003) Redox Sensing and Signalling Associated with Reactive Oxygen in Chloroplasts, Peroxisomes and Mitochondria. Physiological Plantarum, 119, 355-364. https://doi.org/10.1034/j.1399-3054.2003.00223.x

[40] Phang, T., Shao, G. and Lam, H. (2008) Salt Tolerance in Soybean. Journal of Integrative Plant Biology, 50, 1196-1212. https://doi.org/10.1111/j.1744-7909.2008.00760.x

[41] Yoshiyama, K.O., Kimura, S., Maki, H., Britt, A.B. and Umeda, M. (2014) The Role of SOG1, a Plant-Specific Transcriptional Regulator, in the DNA Damage Response. 
Plant Signaling \& Behavior, 9, e28889. https://doi.org/10.4161/psb.28889

[42] Sajedi, N.A., Ardakani, M.R., Rejali, F., Mohabbati, F. and Miransari, M. (2010) Yield and Yield Components of Hybrid Corn (Zea mays L.) as Affected by Mycorrhizal Symbiosis and Zinc Sulfate under Drought Stress. Physiology and Molecular Biology of Plants, 16, 343-351. https://doi.org/10.1007/s12298-010-0035-5

[43] Sajedi, N.A., Ardakani, M.R., Madani, H., Naderi, A. and Miransari, M. (2011) The Effects of Selenium and Other Micronutrients on the Antioxidant Activities and Yield of Corn (Zea mays L.) under Drought Stress. Physiology and Molecular Biology of Plants, 17, 215-222. https://doi.org/10.1007/s12298-011-0067-5 